Messrs. Weiss, and its application is as simple as it has been gratifying.

Fifth stage, usually termed " the toilette of the wound," includes much more than might be presumed from its designation. Not simply is it concerned in clearing the edges of the incision from blood and débris, but also freeing the area of the pupil from any soft lenticular matter that is apt to remain, and give rise to secondary cataract. Various expedients are employed to remove these particles of cortical matter. Great reliance is placed upon digital or instrumental pressure applied either directly to the cornea, or from the outside of the lid. A grave objection to this procedure exists in the fact that even when it is most delicately conducted a rupture of the hyaloid not infrequently occurs, and escape of vitreous follows ; besides, it is often ineffectual. To Dr. McKeown of Belfast belongs the credit of introducing a novel and most effective method of clearing away cortex - viz., by introducing fluid into the capsule of the lens. He published his first series of cases in 1884. The instruments he recommended for injection of the fluid were hollow needles and syringes. Early in 1886 I used McKeown's syringe on two occasions, and these sufficed to show me that, whilst we had a most useful line of practice indicated, the means used for carrying it out were unsatisfactory. Accordingly I replaced the jerky and unsteady flow of the syringe by the equable and controllable flow obtained by a very simple irrigation apparatus. This was fully described at the Brighton meeting of the British Medical Association in 1886. Since then I have continued to use the same simple apparatus, having, however, slightly modified the terminal. The accompanying figure shows the one now in use. I have employed irrigation on more than thirty occasions, always with satisfaction and, I believe, with benefit. The thorough-
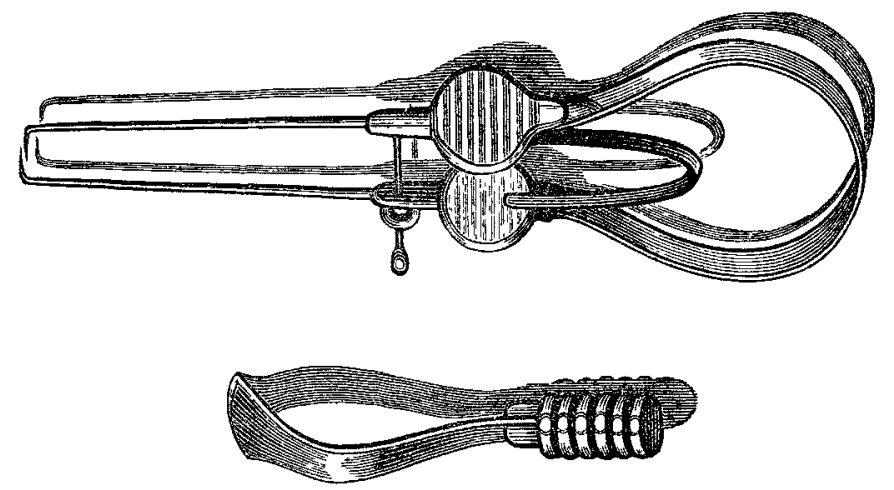

ness with which the irrigator clears away the soft matter is really remarkable. Having by this method secured a black coloboma, and the patient being able to count fingers held in front of the operated eye, it only remained to clear the conjuctival sac from any débris of blood \&c. The outside of the eyelids are usually dredged with iodoform powder, and double pads of Gamgee's lint secured over each eye with strips of plaster. Finally, a roller bandage is fixed over all, the patient removed to bed, where he remains till the eighth day. Many ophthalmic surgeons do not disturb the dressings for a week, unless warned by pain that something is amiss; but as I have seen more than one cornea slough painlessly, it is my custom to remove the pads the day after the operation and replace them by clean ones; the eyelids need not be opened unless there is some redness, swelling, or discharge visible. Should there be anything to arouse suspicion, the lids are opened and an inspection of the wound is made; if now, or at a later period, there are any signs of sloughing, we irrigate the whole of the anterior chamber with an antiseptic fluid, and believe that by these means we have saved from utter destruction one case at least which had the ostrichlike policy of trusting all was going on right because we could not see the untoward processes that had already commenced would undoubtedly have been destroyed.

The judicious application of antiseptics to ophthalmic surgery has long been aimed at by oculists; at the Liver pool Eye and Ear Infirmary we use a solution of biniodide of mercury known as "panas." The instruments to be employed in the operation are immersed in this solution, and the patient's face, as well as the conjunctival cul-de-sac, are thoroughly laved with it.

In conclusion, I beg to acknowledge the kindness and skill of Mr. H. E. Jones, assistant surgeon to the Liverpool Eye and Ear Infirmary, in making sketches of the instruments. Iiverpool.

\section{ON A CASE OF POISONING BY NICOTINE.}

BY G. STILLINGFLEET JOHNSON, M.R.C.S., F.C.S., DEMONSTRATOR OF CHEMISTRY AT KING'S COLLEGE, LONDON.

A TEMPERATE man, aged seventy-five, was found on a doorstep with his throat bleeding, having thrown from him a bottle, and with a greenish vomit on the ground near him. A paper was in his hand, bearing the words "I did this myself." A knife was also near him. He was taken to King's College Hospital, where the bleeding was found to proceed from a divided external jugular vein, which was at once ligatured. Preparations were then made to empty the stomach, but during attempts to adjust the tube of the stomach-pump, which the man resisted actively, he suddenly died, without convulsions. At the necropsy there were no signs of the action of any irritant poison, and the only abnormality observed in any organ was a slight degree of mitral constriction. This being so, and the amount of blood lost being, in the opinion of the house surgeon, quite insufficient to account for death, the stomach and its contents were preserved, and by order of the coroner, Dr. J. Troutbeck, were brought to me for chemical examination. I received the stomach, tied at the cesophageal and pyloric orifices, also a small piece of small intestine, tied at each end; the whole immersed in spirits of wine in a well-stoppered glass bottle. The alcoholic liquor was reserved for separate examination. The contents of the stomach measured rather more than 2 fluid oz. (60 cubic centimetres). It was a brown grumous fiuid, with a feebly acid reaction to litmus paper. It was easily filtered through paper, the insoluble matter being preserved for further examination. The clear filtrate was tested for poisonous metals with a negative result, but it gave the brown precipitate with a solution of iodine in iodide of potassium, characteristic of a vegetable alkaloid. The clear liquid was now rendered slightly alkaline with ammonia, stirred and set aside for a time to favour precipitation. A flocculent precipitate formed at once after adding the ammonia, but did not appear to increase on standing. The precipitate was now separated by filtration, and well washed with cold water. The clear ammoniacal filtrate was tested for alkaloids by acidulating a small portion with hydrochloric acid and adding a solution of iodine in potassium iodide. The alkaloidal precipitate formed as abundantly as ever. On the other hand, the precipitate produced by addition of ammonia to the fluid from the stomach after solution in hydrochloric acid gave only a slight opalescence with solution of iodine in potassium iodide. These results indicate the existence of a soluble vegetable alkaloid. In order to separate the soluble alkaloid from the other sub. stances in the watery solution the clear ammoniacal filtrate was shaken with chloroform. The chloroform separated fairly well from the watery solution, from which it was drawn off by means of a stoppered funnel, and evaporated cautiously over steam. A yellowish oil remained in the dish, whose odour, especially when the dish was warm, was highly penetrating, stinging the nasal membrane like ammonia, and recalling the odour of a foul tobacco pipe. The weight of this oil obtained from 30 c.c. (= one fluid ounce), or half of the stomach contents, was 3 centigrammes $\left(=\frac{46}{100}\right.$ ths of a grain). The oil dissolved almost entirely in water, only a little resinous matter remaining, and its aqueous solution reaction was intensely alkaline to litmus. The aqueous solution was acidulated with hydrochloric acid, and the acid liquid evaporated to dryness over steam. The residue gave an acid solution to water, so that the base forms a hydrochloride. A portion of the solution of the hydrochloride of the alkaloid was rendered alkaline with potash, and the solution was warmed; a strong tobacco-like odour was developed. A second portion of the solution of the hydrochloride of the alkaloid was mixed with an aqueous solu. tion of mercuric chloride, when immediately a copious white precipitate formed, which dissolved on being warmed, and deposited in well-formed crystals on cooling. This reaction proves the absence of coniine, which gives an amorphous precipitate with $\mathrm{HgCl}_{2}$. The above reactions all indicate that the soluble oily alkaloid is nicotine.

I now turned my attention to the alcoholic liquor in which the stomach had been preserved. The filtered liquid was evaporated to dryness over steam, when a copious solid residue remained. The residue was extracted with acidulated water, and the solution gavestrong indication of the presence G 2 
of alkaloidal matter when tested with the solution of iodine in potassium iodide. The alkaloid was found to be soluble in water, and was extracted from the aqueous solution by chloroform, just as in the case of the contents of the stomach described above. The residue, on evaporating off the chloroform, had precisely the same properties as that from the stomach contents, but the quantity of the oily liquid obtained was far greater-viz., 0.188 gramme $(=2.9$ grains). This residue was converted into oxalate by adding excess of oxalic acid to the aqueous solution and evaporating to dryness. The residue was then boiled with absolute alcohol, which dissolved the oxalate of nicotine; filtered alcoholic solution was evaporated to dryness, the oxalate dissolved in water, the solution rendered alkaline with potash and shaken with chloroform. The chloroform solution yielded on evaporation the pure nicotine, paler in colour than the original oil, but otherwise quite similar in properties. Of this purified alkaloid, 2 centigrammes ( $=\frac{3}{10}$ ths of a grain) were injected in the state of aqueous solution into the pleural cavity of a large rabbit by my friend and colleague Mr. C. J. Martin. Almost instantly the animal was thrown into violent convulsions, during which it turned a complete somersault, and was observed to be in a state of extreme opisthotonos as soon as it reached the ground. This condition was rapidly followed by general muscular paralysis and relaxation, and the breathing stopped within three minutes after the exhibition of the poison. The thorax being opened at once, only fibrillar contractions of the heart were observed. At the adjourned inquest before the deputy coroner, Mr. H. E. Barnes, a relative of the deceased having heard my evidence, and my suggestion founded therefrom, that the deceased might have made an infusion of tobacco in some alcoholic liquor, and taken the mixture, informed the coroner that "in conversation the deceased had told him that that was how he meant to do it," from which it would appear that the man was cognisant of the highly toxic nature of tobacco infusions. As the bottle which the deceased threw away was broken, no clue whatever was given to me as to the nature of the poison. As regards the absence of convulsive movements in this case, such movements appear not to be invariably produced by tobacco intoxication in the human subject. Tetanic convulsions were present in one case recorded by Taylor, but absent in another. Possibly the quantity of the poison taken may account for these different effects, for no convulsion took place in the case observed by Taylor himself, where a man swallowed pure nicotine from $a$ bottle, and died within five minutes.

\section{Climital allotes:}

\section{MEDICAL, SURGICAL, OBSTETRICAL, AND THERAPEUTICAL.}

\section{A CASE OF SPORADIC CRETINISM.}

By Arnallt Jones, L.R.C.P. Loxd., M.R.C.S. Eng.

THE following. notes refer to the case of R. G-, who suffered from sporadic cretinism, and died at the age of two years and $a^{*}$ half, having been under my observation for nearly two years. I was first consulted when the child was ten months old, because the parents were alarmed about the condition of his tongue, which had grown large and unwieldy, protruding constantly through the lips and threatening to interfere with respiration. The child certainly had nacroglossia, but he also had a cretinoid appearance. When seven months old he was able to stand upon his legs and also to articulate "dadda" and similar nursery calls, and he took an interest in things that went on around him. When nine months old, however, he commenced to have violent fits of crying, and a blight seemed to come over the child. The tongue now started growing till it protruded through the lips, keeping the mouth constantly wide open. After he reached the age of nine months he ceased to grow, and his appearance became very striking. The hair was coarse and scanty; the skin thickenea, rough, and scaly in parts, the forehead shallow and face wide, the nose broad and flat, the lips thick, with a wide open mouth constantly dribbling saliva, while the features were utterly devoid of expression; the hands were broad and rough skinned, and the fingers stumpy. No trace of the thyroid gland could be made ont, but there was a mass of fat in each supra-clavicular triangle. Some stress was laid by the late Dr. Hilton Fagge upon the presence of these collections of adipose tissue in this situation, and in this particular case these fat collections were at one time nearly the size of a hen's egg, but during the last few weeks of the patient's existence they dwindled down to the size of a pigeon's egg. The teeth were late in appearing; they were friable and ill-formed and crumbled away early. The child was subject to violent fits of crying ; these came on at varying intervals, and if he once started he would go on perhaps for hours at a stretcb. Otherwise, he accepted his fate in a placid, indifferentmanner. Threedays beforehis death violent coughing came on with hæmoptysis. The child died suddenly in convulsions, and, unfortunately, this event occurred during my absence on a holiday; consequently a necropsy was not obtained. The actual cause of the disease in this case is difficult to ascertain. The water drunk by the family was not impregnated with the salts of lime or magnesia; their house is built on pennant rock (sandstone), which here is some hundreds of feet in thickness; there was no consanguinity on the part of the parents, and no specific history on either side. The father was addicted to intemperance at times, a fact which would carry some weight with those who accept a theory as to the etiology of sporadic cretinism suggested by Dr. Langdon Down. This disease has been noticed more than once in several children of the same parents; and here that is likely to be the case, as a younger child of this family is at the present time developing some of the symptoms of incipient cretinism.

Aberavon, Glamorgan.

\section{XANTHOMA MULTIPLEX.}

\section{By G. C. StePHen, L. R.C.P. LoNd.}

ON consulting Dr. Crocker's book on Diseases of the Skin, I find that only forty cases of xanthoma multiplex occurring after puberty have been recorded. I therefore trust the following notes of the case of a patient who recently consulted me for brow neuralgia and jaundice, associated with that interesting affection of the skin xanthoma multiplex, may prove of interest.

Miss - , aged sixty, states that she had always enjoyed fairly good health until from two to three years ago, when she suffered a good deal from bronchial and nasal catarrh and diarrhœea ; but from these she appeared to havequite recovered until she was taken ill in November last with supra-orbital neuralgia and erysipelas of the right side of the face. In January, patches of xanthoma appeared on the hands, feet, wrists, and face, followed in a short time by very intense jaundice. As the patient was at this time residing in a malarious district of America, I think the jaundice was attributable to the malarial cachexia there acquired. When I first saw her, May 26th, 1890, there were numerous buffcoloured patches on the palms and backs of the hands and wrists, and in the flexures of the elbows and knees; also on the face there were several patches not raised above the level of the skin. The lesions on the hands and feet were from about the size of a grain of wheat to that of a threepenny piece. Some were perfectly flat, and on passing the tingers over them no protuberance was noticeable (xanthoma planum). Others, however, were slightly raised above the level of the skin (xanthoma tuberosum). The subjective symptoms were severe itehing, particularly at the time of the formation of new lesions. I have repeatedly examined the urine without discovering any trace of sugar. Under antimalarial treatment, and drachm doses of phosphate of soda three times a day, the jaundice has improved very much, and the irritation of the skin and neuralgia have almost entirely disappeared. The patient feels confident that some of the patches of xanthoma have disappeared, but I cannot say that I have noticed more improvement than that they have become less raised above the level of the skin. The patient's statementis no doubt important, however, in view of the fact that Mr. Jonathan Hutchinson, in a recent number of the Archives of Surgery, reports a case with symptoms very similar to those of my patient, in which xanthoma dis. beticorum was suspected from the irritating character of the 